P170 (continued)

(24\%). The analysis revealed that the materials often contained inaccurate or conflicting information when compared to national recommendations, were on a reading level greater than the 5 th grade $(76 \%)$, lacked cultural appropriate (61\%) or relevant images (53\%), were text or topic dense and, lacked easy to read font (51\%). Approximately $40 \%$ or more of the materials were not specific about children ages B-24 unless it was a developmental handout.

Conclusions and Implications: Future nutrition education efforts should consider developing simple, evidence-based, culturally appropriate outreach materials on a lower reading level for parents of children B-24 to prevent early childhood obesity.

Funding: USDA, The Child Health and Development Institute of Connecticut, Inc.

\section{P171 Exploring Mobile Device Use among Preschoolers: A Feasible Intervention Strategy for Rural Families?}

Cris Benz, MS, RD, Colorado State University; Morgan McCloskey, MSPH; Susan L. Johnson, PhD, University of Colorado Anschutz Medical Campus; Lauren Clark, PhD, University of Utah; Darcy Thompson, $M P H, M D$, University of Colorado Anschutz Medical Campus; Barbara Chamberlin, PhD, New Mexico State University; Laura Bellows, PhD, MPH, RD, laura.bellows@colostate.edu, Colorado State University, 1571 Campus Delivery, Fort Collins, CO 80523

Objective: This study aimed to elicit input from Head Start/preschool parents on child mobile device use to inform nutrition and physical activity intervention strategies for families in rural Colorado.

Study Design, Setting, Participants: Semi-structured telephone interviews were conducted to explore parental beliefs, opinions and rules regarding child mobile device use (e.g. smartphones, tablets); content, frequency and purpose of child device use; and parental opinions regarding the feasibility of using mobile devices and applications (apps) to deliver nutrition and physical activity education for preschoolers.

Outcome Measures: Interviews were recorded and transcribed verbatim, and data were content analyzed into categorical responses to research questions.

Results: Parents $(n=29)$ were $93 \%$ mothers, 38\% Hispanic, $41 \%$ with high school degree, and 65\% low-income. Most parents co-used mobile devices with their preschooler. Parents reported concerns about potential overuse of these devices, yet viewed their child's use as positive for building school-readiness skills. Parents use devices for behavior management and most have specific rules tied to using entertainment-related apps. Using apps to encourage child physical activity was favored by parents, but they found it difficult to envision how apps could improve children's nutrition and feeding behaviors. The idea of children using devices to be ready for a technology-driven world appealed to parents, particularly in balance with traditional play.

Conclusions and Implications: Parents of preschoolers in rural Colorado respond favorably to the concept of mobile apps to improve children's school readiness and to promote physical activity. Developers of mobile apps will be well served to integrate school readiness skills (e.g. vocabulary, counting, etc.) into app development.

Funding: USDA

\section{P172 Families Eating Smart Moving More: Development of a Program Assistant Guide for the Head Start Audience}

Kelly Godwin, BS, East Carolina University; Lorelei Jones, MEd, North Carolina State University; Susan Chase, MEd; Virginia C. Stage, PhD, LDN, RDN, carrawaystagev@ecu.edu, East Carolina University, Health Sciences Building 4310J, Greenville, NC 27834

Objective: Development of an evidence-based teaching guide for Expanded Food and Nutrition Education Program (EFNEP) Program Assistants (PAs) in North Carolina (NC). This resource provides guidance to help PAs adapt an existing curriculum, Families Eating Smart \& Moving More, to educate Head Start (HS) teachers on positive health behaviors.

Theory, Prior Research, Rationale: Classroom nutrition education can improve children's eating habits and long-term weight status, making teachers important partners for encouraging healthy lifestyles. However, HS teachers may not be prepared to serve as role models for children due to their limited knowledge. Professional development opportunities targeting teacher health behaviors are needed.

Description: Target audience were Head Start teachers in NC. The guide presents EFNEP PAs with guidance for educating HS teachers including planning considerations, solutions to potential barriers, and classroom tips for making nutrition education more adaptable to HS audience.

Method: PAs delivered the 6-lesson series to HS teachers in seven NC centers to provide context to the guide's development. After implementation, two HS nutrition coordinators and 30 teachers in seven ENC centers provided feedback on how to best implement the program in the HS environment. Data was collected in the form of interviews and teacher journals.

Conclusion and Implications: EFNEP will use the guide to train PAs in other counties to help HS teachers improve their personal nutrition and physical activityrelated health behaviors. Future research will explore teachers' efficacy to implement more nutrition education in their classrooms after participating in Families Eating Smart, Moving More.

Funding: East Carolina University Office of Public Service and Community Relations

\section{P173 WITHDRAWN}

